\title{
Mediation of T-Cell Activation by Actin Meshworks
}

\author{
Peter Beemiller and Matthew F. Krummel \\ Department of Pathology, University of California, San Francisco, San Francisco, California 94143-0511 \\ Correspondence: matthew.krummel@ucsf.edu
}

Although the actin cytoskeleton and T-cell receptor (TCR) signaling complexes are seemingly distinct molecular structures, they are tightly integrated in T cells. The signaling pathways initiated by TCRs binding to peptide MHC complexes are extensively influenced by the actin cytoskeletal activities of the motile phase before TCR signaling, the signalosome scaffolding function of the cytoskeleton, and the translocation of signaling clusters that precedes the termination of signaling at these complexes. As these three successive phases constitute essentially all the steps consequent to immune synapse formation, it has become clear that the substantial physical forces and signaling interactions generated by the actin cytoskeleton dominate the signaling life cycle of TCR signalosomes. We discuss the contributions of the actin cytoskeleton to TCR signaling phases and model some remaining questions about how specific cytoskeletal factors regulate TCR signaling outcomes.

$\mathrm{T}_{\mathrm{i}, \mathrm{s}}$ he activation of $\mathrm{T}$ cells is controlled primarily by T-cell receptors (TCRs) interacting with peptide-loaded major histocompatibility complexes (pMHCs) as T cells scan the surface of antigen presenting cells (APCs). Because T cells are continuously motile cells that transit through lymph nodes in their surveillance, it is clear that TCR triggering must occur within the context of physical forces that might rapidly separate TCRs from agonist pMHCs. Moreover, crawling $\mathrm{T}$ cells do not truly come to rest at the surfaces of APCs following TCR engagement. Instead, they continuously extend protrusions over APCs and move along the surface of their partner (Gunzer et al. 2000). In their initial encounters with antigen-bearing dendritic cells (DCs), T cells also often rapidly couple and uncouple on the order of minutes, rather than dwelling for extended periods of time on single DCs (Gunzer et al. 2000; Mempel et al. 2004). This dynamic coupling allows $\mathrm{T}$ cells to quickly sample a large proportion of the total APC membrane pool in search of their cognate antigen. Still, these transient contacts are productive-they induce calcium fluxes and the expression of markers of activated $\mathrm{T}$ cellsindicating that TCR signalosome outputs can be initiated in mere minutes and survive the dissolution of contacts, even under the mechanical stress of cytoskeletal remodeling.

TCR signaling requires the dynamic recruitment of a macromolecular complex of kinases, scaffolding molecules, and other signaling effectors to a triggered TCR. Assembly of this macromolecular signaling complex must be very sensitive and occur rapidly, or there is a

Editors: Lawrence E. Samelson and Andrey Shaw

Additional Perspectives on Immunoreceptor Signaling available at www.cshperspectives.org

Copyright (C) 2010 Cold Spring Harbor Laboratory Press; all rights reserved; doi: 10.1101/cshperspect.a002444

Cite this article as Cold Spring Harb Perspect Biol 2010;2:a002444 


\section{P. Beemiller and M.F. Krummel}

\section{A}

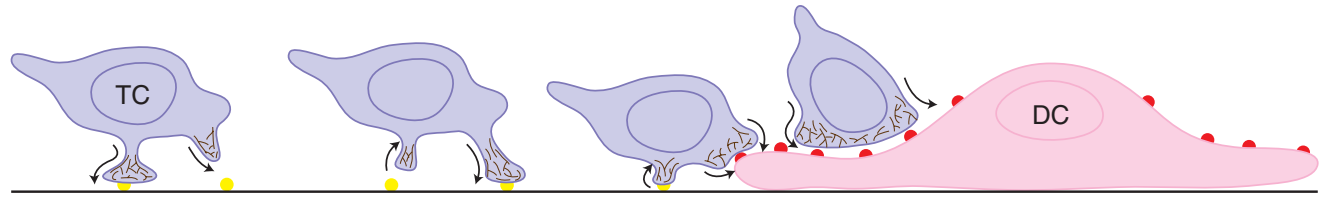

B
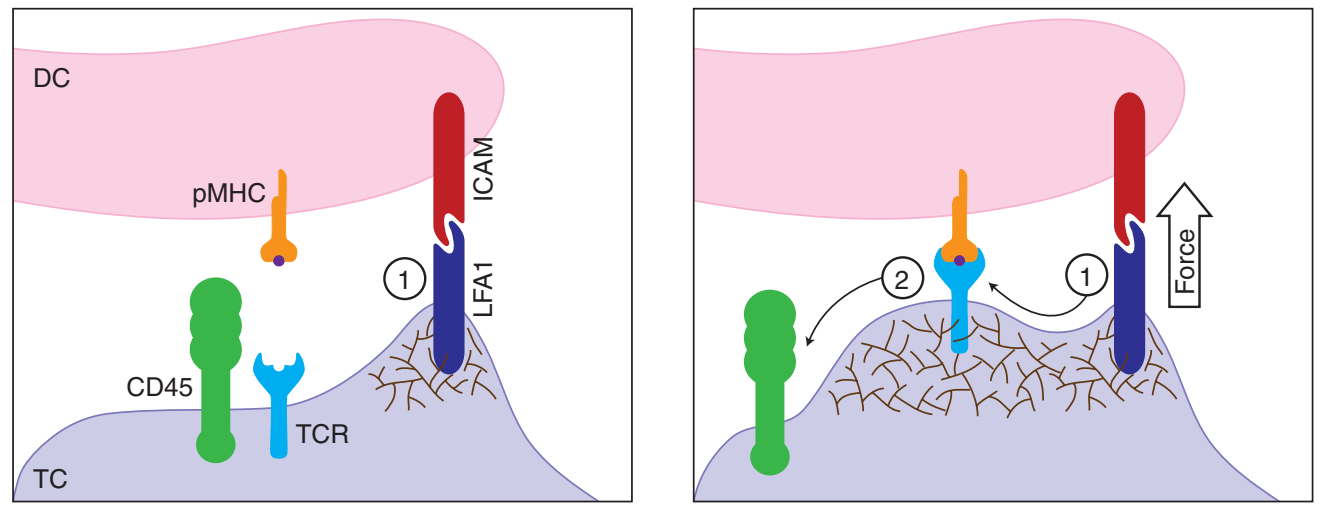

C

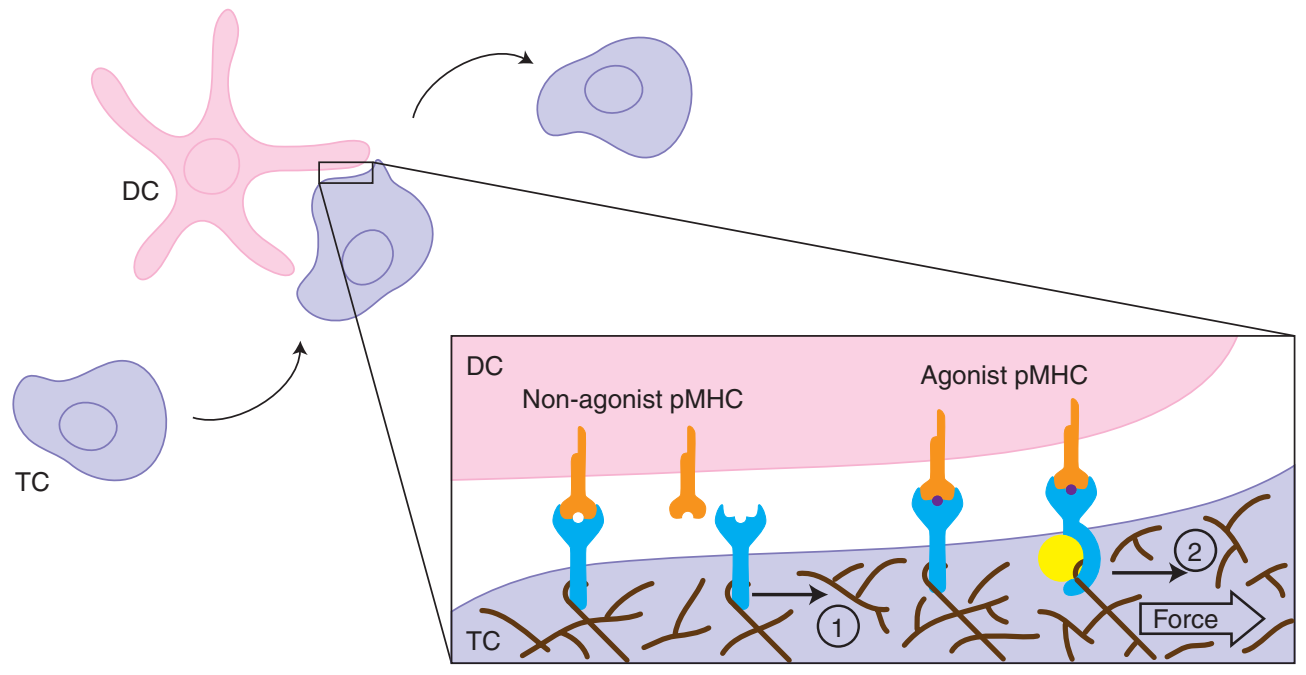

Figure 1. The actin meshwork of the motile T cell establishes a mechanical regime of TCR triggering. $(A) \mathrm{T}$ cells (TC) display various modes of motility depending on the adhesive properties of their microenvironment. To achieve high velocities, T cells create series of small, short-lived contacts with substrates. The pseudopod sequences are generated by cycles of actin polymerization followed by myosin generated contractility. When $\mathrm{T}$ cells encounter the adhesion promoting surface of an APC, such as a dendritic cell (DC), integrin ligation causes the $\mathrm{T}$ cells to spread more extensively, maximizing surface interactions between the cells. Cell body motion does not completely stop, though, and T cells continue to drift over the surface of the APC as they scan for their cognate pMHC. (B) The actin cytoskeletal remodeling induced by integrin ligation during T-APC coupling assists TCR-pMHC interactions. Branched actin network formation (brown), initiated from ligated LFA1 (purple), presses the cell surfaces together (1) to a length scale that allows pMHC (gold) binding to TCRs (blue). (See facing page for legend.) 
risk that the TCR will release the pMHC ligand, and the $\mathrm{T}$ cell will fail to register the antigen hit. Conversely, the signalosome assembly mechanism needs to discriminate against TCRs interacting transiently with a vast array of pMHCs presenting nonagonist peptides. Viewed in this manner, a scheme that rapidly dissociates TCRs from MHCs loaded with endogenous peptide, freeing them to rebind and test other MHCs, is desirable. It is notable that several TCR signaling factors carry binding sites for actin binding proteins or actin itself (Rozdzial et al. 1995; Zhang et al. 1999; Zeng et al. 2003; Phee et al. 2005; Gomez et al. 2006). Through these actinassociated factors, agonist-triggered TCRs rapidly assemble stabilized signaling platforms that survive mechanical disruption.

In concert with adhesive integrin interactions and costimulatory receptor signaling, TCRs orchestrate a reorganization of the T-cell plasma membrane that may begin with a handful of receptors and eventually encompasses the entire contact face with the APC (some $50-100 \mu \mathrm{m}^{2}$ ). TCRs first aggregate into micron scale clusters of TCRs, then flow to the center of the contact face, generating the central supramolecular activating complex (cSMAC) of the immune synapse (Monks et al. 1998; Grakoui et al. 1999; Krummel et al. 2000). Underscoring the importance of the cytoskeleton, the actin depolymerizing toxins latrunculin A and cytochalasin D are potent inhibitors of T-cell activation and block both TCR microcluster formation and cSMAC coalescence (Wulfing et al. 1998; Grakoui et al. 1999; Krummel et al. 2000; Varma et al. 2006). Ultimately, it is the coordination of the local interactions between receptors and effectors with the cell morphological level rearrangements that determines the nature and magnitude of $\mathrm{T}$-cell responses to pathogens. Regulation of TCR signaling lifecycles and T-cell responses, therefore, falls squarely on the actin cytoskeleton.

\section{TRIGGERING}

TCR triggering occurs on interstitially motile $\mathrm{T}$ cells undergoing constant morphological remodeling and crawling at speeds of approximately $10 \mu \mathrm{m} / \mathrm{min}$ (Miller et al. 2002). In vitro, the actinomyosin cytoskeleton reacts to local integrin levels to allow $\mathrm{T}$ cells to adopt one of two crawling modes (Jacobelli et al. 2009). In the faster "walking" mode, T cells create relatively small contacts to grab surfaces and bound from one contact to the next (Fig. 1A). In a mesenchymal mode, $\mathrm{T}$ cells place large portions of their membrane into contact with the surfaces they are surveying. These large, contiguous contacts translate as the $\mathrm{T}$ cell moves forward, giving rise to a "moving walkway" of T-cell membrane that enables extensive scanning of the underlying surface. Although these modes are only presumed to occur in vivo, recent evidence indicates that $\mathrm{T}$ cells employ a "millipede" type motility to move along the blood vessel endothelium that is similar to the walking mode seen in vitro (Shulman et al. 2009). The need for the fast mode may be most pronounced when navigating between loose adherence zones on follicular reticular cell fibers in the lymph nodes, which $\mathrm{T}$ cells appear to use to help guide their circuit from afferent to efferent vessels (Bajenoff et al. 2008). Furthermore, transitions between modes are hinted at by the variety of cell shapes and speeds observed within the T-cell zone (Miller et al. 2002). The actin cytoskeletal mechanics of these motility modes are an essential parameter for TCR triggering, because TCR-pMHC ligation logically

Figure 1. (Continued) By pressing the cell membranes into close proximity, actin polymerization drives bulky, inhibitory phosphatases, such as CD45 (green), from the zone of pMHC-TCR ligation (2). (C) T-cell motility places continuous stress of molecules interaction across the T cell-APC junction. In the receptor deformation model, actin cytoskeletal movements introduce shear force that can break TCR-pMHC interactions. When a perpendicular force is applied (1), TCRs bound to pMHCs that are not bearing the T cells agonist ligand (left) rupture, freeing the TCR to scan another pMHC. When a TCR binds a pMHC bearing the agonist ligand (purple, at right), shear force (2) induces a conformational change, triggering the receptor and recruitment of signalosome factors (yellow). 
requires tight juxtaposition between domains on the T-cell surface that bear TCRs and membrane regions on the APC that display agonist pMHC complexes. By establishing the rate of T-cell scanning across APC membranes and the extent of the T cell-APC contact area, the actin cytoskeleton dictates the time window in which a TCR will bind a specific pMHC complex.

In addition to establishing the transience of membrane-membrane interactions, the cytoskeleton also facilitates T cells' exceptional responsiveness to TCR engagement. From the host perspective, it is undesirable for $\mathrm{T}$ cells to fail to activate following encounters with antigens, no matter how limiting the dose. TCRs, therefore, must be very sensitive- and there is no doubt that they are sensitive: CD8-positive T cells are potentially capable of responding to a single agonist peptide (Sykulev et al. 1996), whereas 1-10 pMHCs can activate CD4 T cells thanks to their coreceptor (Irvine et al. 2002). At a naive glance, the ideal mechanism to achieve such sensitivity might seem obvious: A receptor should combine a very fast association rate with a very slow dissociation rate for agonist ligands. Studying the measured binding and unbinding kinetics of some model TCRs quickly shows that not to be the case for TCRs (Davis et al. 2003). It appears that $\mathrm{T}$ cells instead take advantage of rapid unbinding of pMHCs to sequentially trigger multiple TCRs, (Valitutti et al. 1995; Lanzavecchia et al. 1999). In effect, the successive ligation of TCRs serves as a gain, turning each pMHC into many activated receptors. The development of mathematical models has helped to explain how $\mathrm{T}$ cells have adapted to TCR biochemical properties to achieve both fidelity and sensitivity (McKeithan 1995; Coombs et al. 2002; Wedagedera and Burroughs 2006).

But what sort of biomechanical processes can we imagine that would facilitate this mechanism of triggering TCRs? Conformational change models of TCR triggering fell into disfavor because of an apparent lack of structural data to support large scale structural alterations following pMHC binding (Ding et al. 1999; Willcox et al. 1999; Degano et al. 2000). However, evidence for rearrangements between members of the TCR complex or within various TCR subunits has been described (Krogsgaard et al. 2003; Kim et al. 2009). These studies provide clues to how information about the TCR binding partner is transmitted across the $\mathrm{T}$ cell membrane, but don't immediately address how the TCR translates this into a signaling output. Under the kinetic-segregation model, $\mathrm{T}$ cells use the intercellular spacing to exclude inhibitory molecules from the domain of triggered TCRs (van der Merwe et al. 2000). By creating zones of close contact in which TCRs can bind to pMHCs, integral membrane phosphatases with large ectodomains that antagonize TCR phosphorylation can be excluded. Specificity is achieved because relatively weak interactions, such as between TCRs and self-peptide MHCs, dissociate more rapidly, and the phosphorylated TCRs diffuse out of the close contact zone. This allows inhibitory phosphatase activities to deactivate the released TCRs. In contrast, relatively stable TCR-pMHC interactions would tether the TCR in the zone of tight contact longer, providing a window to assemble an activated, phosphatase-resistant signalosome. Although lipid rafts might explain how TCRs and other prosignaling factors remain tethered within the area of close contact, it is not clear how the weak adhesive strength from a limited number of pMHC-TCR interactions could maintain a closely spaced junction between the two cellular membranes at the size scale required for the kinetic-segregation model (Burroughs et al. 2006). To this end, mechanical pressure exerted by the actin cytoskeleton of the leading edge may be instrumental in pressing the apposed cell membranes together, squeezing proteins with larger ectodomains away from the tightest contact (Fig. 1B). Consistent with this idea, increasing the length of the pMHC extracellular domain impacted TCR segregation from CD45 and antagonized receptor triggering (Choudhuri et al. 2005). Kinetic-segregation might partially explain why integrin-mediated adhesion enhances TCR signaling even on bilayer systems (Grakoui et al. 1999; Porter et al. 2002; Suzuki et al. 2007), as the longer LFA1ICAM pairs would establish zones of looser contact to accommodate bulky phosphatases. 
It is important to note that one of the phosphatases associated with Lck inhibition and TCR deactivation, CD45, also appears to positively regulate Lck by dephosphorylating an inhibitory phosphotyrosine of Lck (Biffen et al. 1994; Stone et al. 1997). As a result, it would appear that CD45 must have access to TCR signalosomes near the time of triggering; otherwise, phosphorylation of the Lck inhibitory site could inactivate Lck and cause a triggering failure. Balancing the activating and inhibitory potential of CD45, then, may come down to timing access and exclusion through actinmediated force generation. Following dephosphorylation of the inhibitory site of Lck, CD45 might be rapidly driven from the zone of close contact as integrin-mediated actin polymerization presses the cell membranes together, preventing it from dephosphorylating the active site phosphotyrosine of Lck (Thomas and Brown 1999). Spatiotemporal analysis of CD45 at T-APC interfaces indicated that CD45 was colocalized with Lck in young cSMACs and segregated from Lck at more mature interfaces (Freiberg et al. 2002). So far, though, only limited evidence for the sort of microscale exclusion of CD45 envisioned by the kineticsegregation model has been presented (Choudhuri et al. 2005; Choudhuri et al. 2009). Furthermore, the importance of the extracellular domain to signaling is not obvious, and, in fact, smaller extracellular domains of CD45 appear to positively correlate with coreceptor association and antigen receptor signaling (Leitenberg et al. 1996; Shenoi et al. 1999; Trowbridge and Thomas 2003). Therefore, a variation on this apposition-induced triggering mechanism could be that the force pressing the membranes together induces the bending of TCR complexes in TCR-pMHC pairs, revealing sites on CD3 for phosphorylation. Weaker, nonagonist pMHC-TCR interactions would release before phosphorylation sites expose, allowing the TCR to relax into the untriggered, resting conformation.

$\mathrm{Ma}$ and others recently proposed a very intriguing modified conformational change model (Ma et al. 2008) hypothesizing that actin cytoskeleton generated shear force parallel to the synapse, rather than a force perpendicular to TCRs, facilitates receptor triggering. In this receptor deformation model, they argue that the stress on TCR-pMHC pairs induced by spreading over an APC essentially analyzes TCRpMHCs interactions: Strong agonists provide a sufficiently high resistance to bond rupture to induce a deformation of the TCR upon actin cytoskeletal induced stress. That deformation is interpreted as an authentic antigen encounter only if the deformation is large enough or sufficiently persistent to lead to signalosome assembly on a deformed TCR (Fig. $1 \mathrm{C})$. This hypothesis is particularly interesting when viewed in the context of a mixture of integrin and TCR signaling. As the T cells crawl over an APC, LFA-1 stimulation will be temporally coincident with initial TCR-pMHC ligation. The background of integrin signaling can facilitate dynamic actin cytoskeletal polymerization that will generate the shear force needed to test recently bound TCRs. As a result, this model lends itself to a very elegant mechanical explanation of why integrin cosignaling is required to sustain TCR signaling-beyond the adhesion strength supplied to hold the T-APC couple together, it drives the shear induced bond rupture testing with actin remodeling (Fig. 1C). This fits nicely with observations from Takashi Saito's group. They found that LFA1 signaling induced an actin cloud at the center of contact sites between T cells and APCs in the absence of antigen (Suzuki et al. 2007). The formation of this cloud lowered the threshold for T-cell activation after subsequent antigen exposure.

It is not clear if the sheer-induced TCRpMHC bond testing would require the assembly of a nascent signalosome with signaling factors providing specific binding sites for actin filaments. The cytoskeleton could exert force on a TCR without a specific molecular coupling linking receptors to filaments through general steric interactions. By first assembling a nascent actin-linked signalosome around the receptor, though, the cytoskeleton could apply force to the TCR-pMHC bond no matter what direction the filament moved relative to the TCR. If the receptor deformation testing mechanism is 
engaged after the assembly of an initial signalosome, a secondary proof-reading mechanism is needed to explain how the signalosomes of TCRs bound to weak agonists, which fail the sheer test, can be reverted. SHP-1 phosphatase regulates a negative feedback loop that deactivates TCR signaling initiated by weak agonists, whereas ERK can stabilize Lck modification and stabilize signalosomes (Stefanova et al. 2003). Combining this observation with the receptor deformation model, ERK may be recruited specifically to those receptors that have undergone deformation, exposing a binding site for ERK or an ERK-binding intermediate.

This model can also explain why soluble multivalent, but not monomeric, pMHCs can stimulate T cells (Boniface et al. 1998; Cochran et al. 2000), which is sometimes taken as evidence for a multimerization model of TCR triggering. For single TCRs ligated to soluble pMHCs, the added mass of the bound pMHC is unlikely to distort the TCR conformation under a pulling force applied by actin. When two pMHCs are bound to nearby TCRs, though, small deviations in the directions or magnitudes of the forces applied to the TCRs could induce conformation changes in one or both receptors. This model might even be able to explain the "pseudodimer" mechanism (Krogsgaard et al. 2005), if the added stability of the low-affinity member of the pseudodimer can increase the rupture strength of the high-affinity counterpart just enough to induce a conformational change.

An intriguing recent observation indicates that the interaction of untriggered TCRs with the actin cytoskeleton increases in response to calcium release (Dushek et al. 2008). In T cells stimulated to release calcium with ionomycin, rather than antigenic stimulation of TCRs, F-actin increased and TCR mobility decreased. Although the decrease in TCR mobility could be described by diffusion trapping, the authors argue that a model combining diffusion trapping with receptor-actin binding better explained the observed changes in TCR mobility. Even if we dismiss the actin binding component, diffusion trapping alone of untriggered TCRs has significant implications for the biology TCRs.
Migrating T cells are most sensitive to TCR triggering at the leading edge (Negulescu et al. 1996), site of the filamentous actin-rich lamellipod and lamella (Pollard and Borisy 2003). Trapping TCRs might prepare them for signalosome assembly by placing them in proximity to a number of effectors of TCR signaling, or facilitate formation of the cytoskeletal linkages that will test pMHC interactions through the receptor deformation mechanism. It would appear, then, that TCR triggering mechanisms are adapted to achieve maximum sensitivity within the micro-environmental context of the actin cytoskeleton of motile $\mathrm{T}$ cells. This suggests an additional benefit for scanning $\mathrm{T}$ cells to continuously slide over APC surfaces - it maintains the dynamic actin meshwork that facilitates TCR triggering. Following triggering, it would be expected that TCR signalosome and microcluster life cycles are likewise coordinated with the morphological rearrangements of continuous motility.

\section{SCAFFOLDING}

With the cytoskeletal forces of motility potentially reducing the lifetime of TCR-pMHC interactions on crawling $\mathrm{T}$ cells to a few seconds or even less (Huppa et al. 2010), a system that facilitates the rapid assembly of a signaling complex around a triggered TCR can greatly enhance the sensitivity of TCRs. For technical reasons, imaging TCR signaling dynamics proves to be much easier on stimulatory surfaces, such as antiCD $3 \epsilon$-coated glass or ICAM- $1 / \mathrm{pMHC}$ laden bilayers. When $\mathrm{T}$ cells contacting these surfaces are imaged, TCR microclusters appear very rapidly. Within seconds, a number of effectors are recruited to TCRs, including Lck, ZAP-70, LAT, and SLP-76 (Bunnell et al. 2002; Ehrlich et al. 2002). In a similar kinetic window, cytoskeletal remodeling factors, such as Vav1, WASP, Cdc42, and Rac, colocalize with triggered receptors (Barda-Saad et al. 2005; TskvitariaFuller et al. 2006). By structurally integrating with receptor signalosomes, the actin cytoskeleton serves as a relatively stable scaffold, which can rapidly localize many signaling proteins to receptors (Fig. 2A). Additionally, in linking the 
A

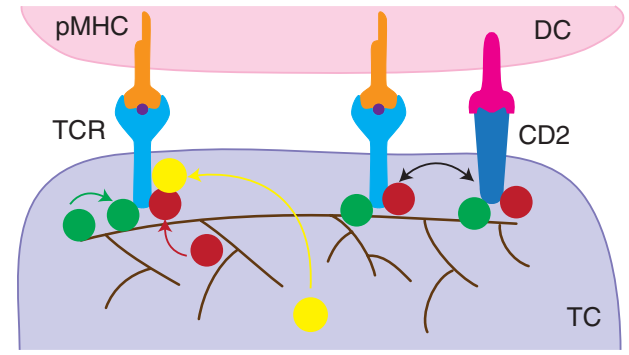

B

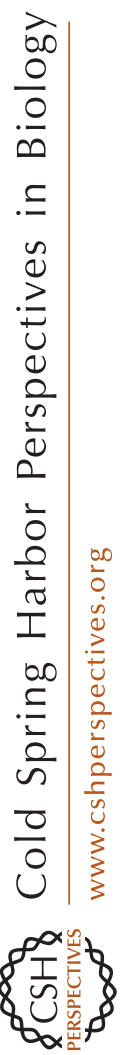

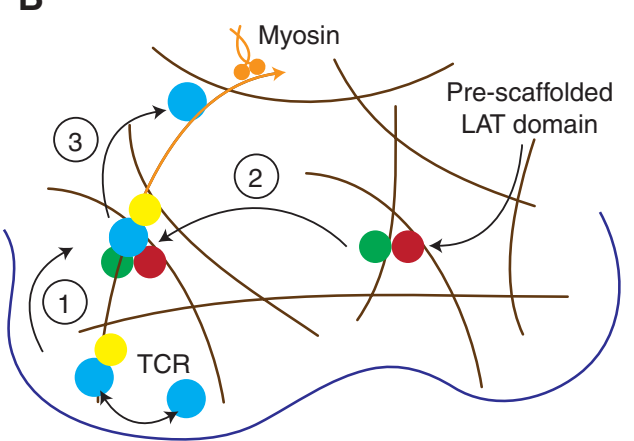

Figure 2. Actin meshwork scaffolding stabilizes signaling clusters and promotes rapid signalosome assembly. (A) Association of TCRs with actin filaments holds receptors in place following triggering, and places the receptors in proximity to actin-associated effectors that can be rapidly recruited to stabilize the triggered TCR on the cytoskeleton (left, red and green). This can increase the lifetime of intermolecular interactions to allow diffusion of soluble effectors (yellow) to signalosomes and microcluster coalescence. Additionally, costimulatory receptors signal through many of the same effectors as TCRs. CD2 has been observed to colocalize with TCRs following stimulation, and can assemble TCR signaling factors into a signalosome resembling a TCR signalosome even before TCR triggering. By sharing effectors, association of CD2 and TCR microdomains allows rapid coalescence of a signalosome around a triggered TCR. (B) TCRs transiently associate with the actin meshwork, either directly or through an intermediate binding partner (yellow). Functional signalosomes can be quickly assembled following receptor triggering by concatenating actin-dependent TCR membrane microdomains (1) with prescaffolded, LATenriched membrane microdomains (2). To terminate signaling, TCRs can be separated from signalosome factors by myosin based actin filament movement (in gold) that pulls TCRs from the signaling supportive periphery into the center of the immune synapse (3).
Mediation of T-Cell Activation by Actin Meshworks

signaling complexes to the relatively stable actin filaments, the cytoskeleton allows signalosomes to survive pMHC unbinding, facilitating T-cell activation by even a small number of agonists. This allows a single pMHC to serially trigger a number of TCRs (Valitutti et al. 1995), without "resetting" the stimulus at each point. Again, this can help T cells to discriminate strong and weak agonists: Strong agonists ligate TCRs long enough to facilitate an actin-linked signaling assembly and once stabilized, the signaling cluster can survive pMHC release, at least temporarily.

The guanine nucleotide exchange factor Vav1 is well-established as a regulator of TCR signaling (Wu et al. 1995; Costello et al. 1999). As an activator of Rho-family GTPases, Vav1 can activate actin cytoskeletal remodeling through a number of pathways and can mediate indirect association with actin filaments through several binding partners. A few of those interactions in particular, though, help to explain how Vav1 quickly integrates TCRs into the cytoskeleton network and catalyzes signalosome assembly. Although Vav proteins typically have a predominantly cytosolic distribution, a fraction of Vav1 was found to be constitutively phosphorylated and associated with the $\mathrm{CD} 3 \zeta$ chain of the TCR complex in resting cells (Valensin et al. 2002). This result was obtained using cell lines, so the constitutively phosphorylated Vav1 was possibly an artifact of a disregulated signaling pathway. TCR signaling, though, is initiated in the context of costimulatory receptor and integrin ligation, which might preactivate a pool of Vav1 that can associate with CD3̧. Salojin et al. reported that $\mathrm{CD} 28$ costimulation increased Zap-70-mediated phosphorylation of Vav1, and increased LAT-Vav1 interactions (Salojin et al. 1999). CD28 and TCR cosegregation into signaling clusters would give TCRs access to costimulatory receptor activated Vav1 proteins (Yokosuka et al. 2008). Through its association with ZAP-70, then, Vav1 is tied to one of the earliest factors recruited to TCR signalosomes (Salojin et al. 1999; Bunnell et al. 2002; Yokosuka et al. 2005).

Although it is intimately associated with actin remodeling, Vav1 does not directly bind actin. A recently discovered interaction in T cells, 
though, explains how Vav1 can load TCRs onto actin filaments (Gomez et al. 2006). Phosphorylation of HS1 by Lck and ZAP-70 stabilizes actin filaments at the synapse and helps maintain Vav localization to synapses. By mediating a linkage between Vav1 and actin, HS1 can immobilize a multimolecular complex consisting of the TCR with CD3 $\zeta$, ZAP-70, Lck, and Vav1 on an actin filament. As noted above, the signaling fate of this complex may be controlled by conformational deformation induced when the associated microfilament shifts and stresses the TCR-pMHC bond. Somewhat surprisingly, it was recently reported that the GEF activity of Vav1 is not necessary for the transduction of TCR signaling (Miletic et al. 2009). This suggests that Vav1 mediated scaffolding onto the actin cytoskeleton is more important for initiating TCR signaling than the cytoskeletal rearrangements induced by Vav1 downstream of its exchange activity (Miletic et al. 2006).

Prescaffolding signaling molecules into membrane microdomains maintained by the actin cytoskeleton can also increase the rate of signalosome activation following TCR triggering. This can reduce signalosome assembly to a few steps, rather than a sequential integration sequence of diffusing factors. Additionally, holding signaling factors in domains exclusive from TCRs provides a safety mechanism to prevent inappropriate signaling complex formation. Work by Mark Davis' and Bridget Wilson's research groups revealed islands of membrane-associated proteins that depended on the integrity of the actin cytoskeleton (Lillemeier et al. 2006). Recently published experiments following up on this approach showed that TCRs and the critical T-cell signaling linker LAT exist in separate membrane-associated protein islands that aggregate, but do not quite mix, following TCR stimulation (Lillemeier et al. 2010). Concatenation of these domains generated functional TCR signaling complexes. This type of segregation might also account for nonmixing populations of $\mathrm{CD} 3$ and Lck revealed previously by single-molecule imaging (Douglass and Vale 2005). The fusion of two actinassociated domains, then, allows the rapid colocalization of the factors needed to construct a complete signalosome, in contrast to a serial diffusion-based mechanism (Fig. 2B). Costimulation receptors, not just TCRs, use actinmediated signalosome assembly to bring together signaling factors, allowing receptors to cooperatively assemble signaling complexes. Even in the absence of TCR triggering, the CD2 coreceptor generated actin-scaffolded microdomains of TCR signaling factors that included Lck, LAT, and the TCR CD3 $\zeta$ chain (Kaizuka et al. 2009). Fusion of these LAT-bearing microdomains with a triggered TCR microdomain would immediately generate a functional TCR signalosome, allowing CD2 to "prefabricate" a TCR signalosome on an actin filament for a triggered receptor (Fig. 2A).

Although the stabilization provided by actin scaffolding is critical to the assembly of TCR signalosomes and microcluster coalescence (Campi et al. 2005), at some point, microclusters of TCRs become auto-stable. Microclusters formed in T cells before treatment with Latrunculin A persist for minutes after addition of the toxin (Varma et al. 2006). The auto-stabilized components of the clusters do not appear to include all the factors necessary for signaling, though, as the Latrunculin challenge terminated calcium fluxes within a minute. This indicates that the scaffolding provided by the actin cytoskeleton is required to maintain the association between TCRs and signaling factors throughout the signaling life cycle of the receptors. The collection of TCRs into the autostabilized fraction of the microclusters has implications for their life cycle on entering cSMACs. TCRs are held in the cSMAC, even in the absence of continued pMHC interactions, and despite the lack of an extensive actin filament network in the cSMAC (Barda-Saad et al. 2005; Varma et al. 2006). This is probably because receptors held in large aggregates cannot significantly diffuse across the barrier imposed by the dense actin meshwork of the peripheral SMAC ( $\mathrm{pSMAC}$ ). An interesting possibility is that TCR microcluster auto-stability serves as a mechanism to keep TCRs from reentering the signaling-conducive pSMAC region and reactivating, while integrins and other receptors that travel to the edge of the cSMAC 
through centripetal flow (discussed later) are presumably free to recycle and continue stimulating adhesion. By imposing a strict dependence on actin cytoskeletal scaffolding for signal propagation and confining extinguished TCRs in a zone that does not support signaling, the actin cytoskeleton establishes a ceiling for TCR signal generation. This may prevent run-away signal propagation and the induction of T-cell apoptosis in response to strong pathogens.

\section{TRANSLOCATION}

In $\mathrm{T}$ cells transferred onto activating lipid bilayers, the organization of receptors into micron-scale signaling clusters is followed within minutes by the radial translocation of clusters to the center of the contact site. Accumulation of individually trafficked clusters to the center leads to the coalescence of cSMACs, as observed in T-APC couples, and originally reported by Kupfer. As in T-APC couples, the LFA1-ICAM pair occupies the periphery of the interface (pSMAC), although it now appears that integrins also undergo centripetal streaming to some extent (Kaizuka et al. 2007). The functional consequences of the large-scale membrane reorganization initiated by TCR signaling now appear to be the termination of signaling and the internalization of receptors (Mossman et al. 2005; Varma et al. 2006). Despite this, the existence, let alone significance, of long-lasting immune synapses with clearly defined cSMACs and pSMACs in vivo is not clear.

In addition to scaffolding the assembly of signalosomes, the actin cytoskeleton orchestrates the sorting of different receptors into the PSMAC and cSMAC domains. At the periphery of the synapse, TCR microcluster centripetal flow is coupled to actin retrograde flow (Fig. 3A), although it is not exactly clear that this cytoskeletal force extends all the way to the cSMAC. The molecular interactions that facilitate this linkage are incompletely described, but it appears to be a frictional coupling mechanism that allows some slippage of microclusters against the actin meshwork (Kaizuka et al. 2007; DeMond et al. 2008). Using highspeed video microscopy to image the dynamics of TCRs and integrins during synapse formation on bilayers, Kaizuka et al. showed that TCRs and integrins formed spatially separate microcluster domains at the earliest observed time points (Kaizuka et al. 2007). Similar to TCRs, the assembly of integrin clusters required actin cytoskeletal integrity. However, unlike mature TCRs microclusters, which achieved a level of actin-independent stability, intregrins clusters disintegrated following latrunculin treatment. The authors provide additional insight by pointing out that although integrin clusters flowed centripetally, they did not enter the actin-depleted cSMAC.

The observation that both integrin and TCR signalosomes couple with retrograde actin flow poses an interesting problem for models of receptor sorting into the cSMAC and pSMAC domains. If both receptor signaling complexes flow inward, what mechanism allows the cell to distinguish and sort signalosomes so that integrin clusters do not reach the central zone but TCR clusters do? An absolute dependence on the actin cytoskeleton for stability explains why the integrin clusters disassemble at the CSMAC, but it does not indicate which property of a cluster confers stability. This issue is particularly troublesome if, indeed, coupling of signalosomes to the actin cytoskeleton is nonspecific. The work of Jay Groves' research group, which has provided extensive insight into the mechanics of cSMAC generation, indicates that the centripetal travel of a signaling cluster is related to its size (Hartman et al. 2009). By generating cross-linked dimers and tetramers of integrins, they show that increasing the size of integrin clusters increases the extent to which clusters centralize. Tetrameric integrins clusters were able to reach the center of interfaces, with the resulting integrin distribution appearing remarkably similar to TCRs in a cSMAC. The group went on to show that when centralizing receptors reached a barrier, the clusters sorted into a size hierarchy-the largest clusters (typically TCR clusters) pressed against the barrier and forced lower total molecular weight integrin clusters to take a more peripheral position (Fig. 3A). Similarly, tetrameric integrin clusters sorted inside dimeric integrin clusters. 
A
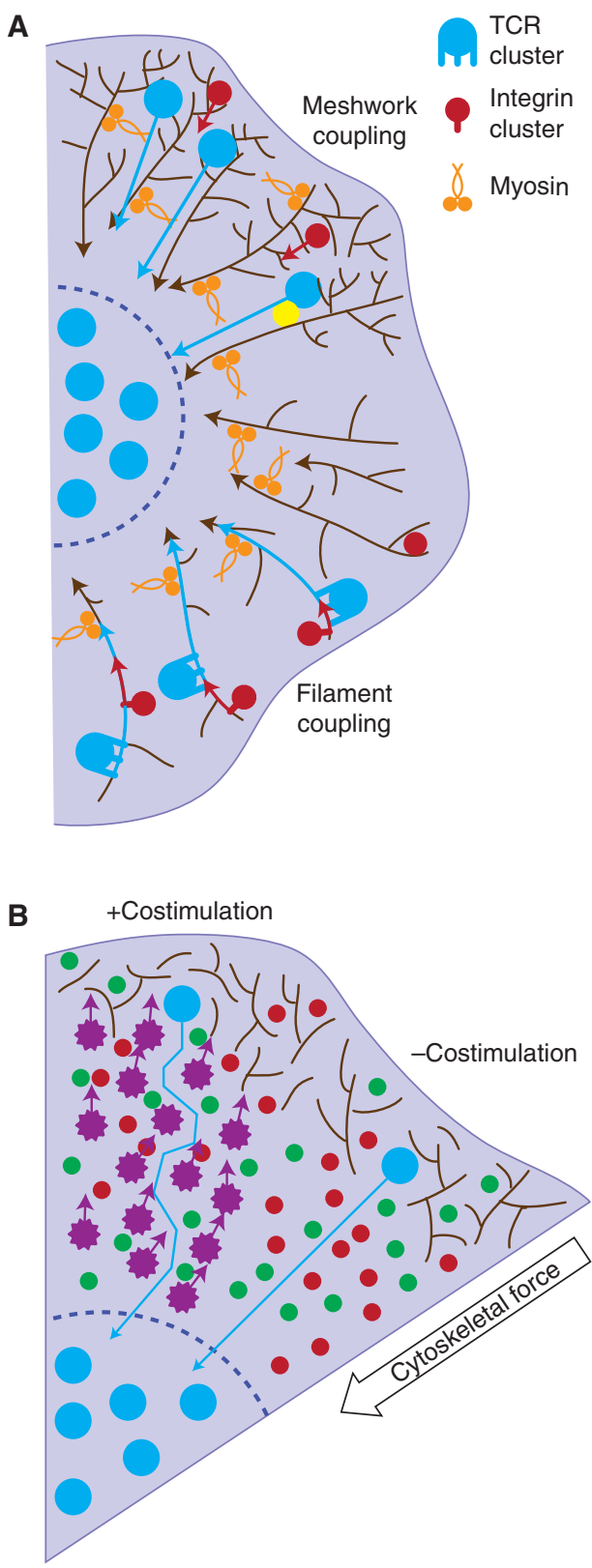

This size-dependent sorting could explain how $\mathrm{T}$ cells modulate TCR signaling to achieve generally comparable outcomes in response to low doses or high doses of antigen: Once clusters reach a certain size (a certain number of TCRs are triggered), a signaling threshold/integral is reached and the clusters flow to the actinpoor central region to be down-regulated. On
Figure 3. Friction applied by the actin meshwork regulates T-cell activation through actin retrograde flow. (A) TCR microclusters (cyan circles) stream centripetally toward the cSMAC boundary (indicated by the dashed purple semi-circle). Receptors couple to retrograde actin flow (brown arrows) generated by actin-associated myosin motor proteins (gold) and by actin polymerization at the periphery of the interface. Because of the density of the actin meshwork in the periphery, receptors need not travel along filaments, but rather can be forced inward nonspecifically (shown at top). Alternatively, differential binding to an actin-associated signaling factor (yellow) could determine the extent to which a receptor centralizes. A generic coupling mechanism is suggested by recent work that indicates that both LFA1 (red circles) and TCRs couple to the centripetal actin flow. The extent to which receptors centralize is controlled by the size of clusters, with larger TCR microclusters consistently sorting to the interior of smaller integrin microclusters (shown at bottom). In a "clutched" mechanism, the larger TCR microclusters (cyan) generate more links to actin filaments than smaller intergrin clusters (red). As a result, TCR signaling clusters show increased centralization. $(B)$ Some costimulation receptors (purple) may function by increasing the time that TCR microclusters spend in the signaling factor enriched periphery of the synapse. By creating a series of frictional barriers (shown at left), ligated costimulation receptors generate forces (purple arrows) that counteract retrograde flow of the cytoskeletal meshwork, increasing the length of the path (cyan line) that microclusters follow to reach the CSMAC. This increases the residence time of the TCR microcluster in the signaling effector enriched pSMAC. In the absence of ligated coreceptors, TCRs quickly pass through the pSMAC, decreasing their residence time in the signal generating region of the synapse (shown at right). 
rates of TCR microcluster growth, increased centralization rates and more rapid attenuation of signaling. Incorporating work from Stephen Bunnell's group, costimulatory receptor effects can be integrated into this model. Nguyen et al. reported that ligation of the VLA-4 integrin reduced the actin-dependent rate of microcluster centralization (Nguyen et al. 2008). They found that this VLA4-mediated effect increased the time that SLP76 remained associated with LAT in active peripheral microclusters (Fig. 3B). Therefore, a major contribution of this kind of costimulation may be to modulate the rate of TCR centralization by increasing friction in the pSMAC, creating a temporary barrier in the periphery (DeMond et al. 2008).

What is not clear yet is the exact nature of the coupling of TCRs to the retrograde actin flow and the molecular motors activities that force receptors to the CSMAC. Myosin generated contractions could pull filaments toward the cSMAC, with TCR microclusters forming stable linkages to filaments. Myosin II was recently implicated in TCR microcluster translocation (Ilani et al. 2009). However, these experiments relied on imaging conditions that can induce blebbistatin crosslinking, the inhibitor used to block myosin II activity, and are at odds with earlier work that found no role for myosin II in synapse stabilization and cSMAC coalescence (Jacobelli et al. 2004). Other motors might also function in the PSMAC to generate flow, but little is known about the distribution of these other myosins in immune synapses. Alternatively, actin polymerization can also generate retrograde flow independently of myosin motor activity (Henson et al. 1999). This could generate centripetal flow of receptors by pushing the actin meshwork and associated receptors away from the cell edge.

As a final note, it should be mentioned that the actin cytoskeleton has roles in immune synapse formation and function beyond regulating the signaling life cycles of TCRs. Centrosome polarization toward the cSMAC depends on the actin cytoskeleton and is critical for directional secretion of granules across the cell-cell interface (Stinchcombe et al. 2006). This process appears to be mediated by formin family actin nucleating factors using aspects of the actin cytoskeleton that are distinct from TCR induced F-actin structures (Gomez et al. 2007). Very recent work indicates that agonist potency and the kinetics of TCR signaling control granule recruitment and cytotoxic $\mathrm{T}$ lymphocyte killing activity (Beal et al. 2009; Jenkins et al. 2009). The actin cytoskeleton, therefore, can also form a structural framework to coordinate TCR signaling life cycles with effector T-cell functions.

\section{REFERENCES}

Bajenoff M, Glaichenhaus N, Germain RN. 2008. Fibroblastic reticular cells guide $\mathrm{T}$ lymphocyte entry into and migration within the splenic T cell zone. J Immunol 181: 3947-3954.

Barda-Saad M, Braiman A, Titerence R, Bunnell SC, Barr VA, Samelson LE. 2005. Dynamic molecular interactions linking the $\mathrm{T}$ cell antigen receptor to the actin cytoskeleton. Nat Immunol 6: 80-89.

Beal AM, Anikeeva N, Varma R, Cameron TO, VasiliverShamis G, Norris PJ, Dustin ML, Sykulev Y. 2009. Kinetics of early $\mathrm{T}$ cell receptor signaling regulate the pathway of lytic granule delivery to the secretory domain. Immunity 31: 632-642.

Biffen M, McMichael-Phillips D, Larson T, Venkitaraman A, Alexander D. 1994. The CD45 tyrosine phosphatase regulates specific pools of antigen receptor-associated p59fyn and CD4-associated p56lck tyrosine in human T-cells. EMBO J 13: 1920-1929.

Boniface JJ, Rabinowitz JD, Wülfing C, Hampl J, Reich Z, Altman JD, Kantor RM, Beeson C, McConnell HM, Davis MM. 1998. Initiation of signal transduction through the $\mathrm{T}$ cell receptor requires the multivalent engagement of peptide/MHC ligands. Immunity 9: 459-466.

Bunnell SC, Hong DI, Kardon JR, Yamazaki T, McGlade CJ, Barr VA, Samelson LE. 2002. T cell receptor ligation induces the formation of dynamically regulated signaling assemblies. J Cell Biol 158: 1263-1275.

Burroughs NJ, Lazic Z, van der Merwe PA. 2006. Ligand detection and discrimination by spatial relocalization: A kinase-phosphatase segregation model of TCR activation. Biophys J 91: 1619-1629.

Campi G, Varma R, Dustin ML. 2005. Actin and agonist MHC-peptide complex-dependent $\mathrm{T}$ cell receptor microclusters as scaffolds for signaling. $J$ Exp Med 202: 1031-1036.

Choudhuri K, Parker M, Milicic A, Cole DK, Shaw MK, Sewell AK, Stewart-Jones G, Dong T, Gould KG, van der Merwe PA. 2009. Peptide-major histocompatibility complex dimensions control proximal kinase-phosphatase balance during T cell activation. J Biol Chem 284: 26096-26105.

Choudhuri K, Wiseman D, Brown MH, Gould K, van der Merwe PA. 2005. T-cell receptor triggering is critically dependent on the dimensions of its peptide-MHC ligand. Nature 436: 578-582. 
P. Beemiller and M.F. Krummel

Cochran JR, Cameron TO, Stern LJ. 2000. The relationship of MHC-peptide binding and $\mathrm{T}$ cell activation probed using chemically defined MHC class II oligomers. Immunity 12: 241-250.

Coombs D, Kalergis AM, Nathenson SG, Wofsy C, Goldstein B. 2002. Activated TCRs remain marked for internalization after dissociation from pMHC. Nat Immunol 3: 926-931.

Costello PS, Walters AE, Mee PJ, Turner M, Reynolds LF, Prisco A, Sarner N, Zamoyska R, Tybulewicz VLJ. 1999. The Rho-family GTP exchange factor Vav is a critical transducer of $\mathrm{T}$ cell receptor signals to the calcium, ERK, and NF-kappa B pathways. Proc Natl Acad Sci 96: 3035-3040.

Davis MM, Krogsgaard M, Huppa JB, Sumen C, Purbhoo MA, Irvine DJ, Wu LC, Ehrlich L. 2003. Dynamics of cell surface molecules during $\mathrm{T}$ cell recognition. Ann Rev Biochem 72: 717-742.

Degano M, Garcia KC, Apostolopoulos V, Rudolph MG Teyton L, Wilson IA. 2000. A functional hot spot for antigen recognition in a superagonist TCR/MHC complex. Immunity 12: 251-261.

DeMond AL, Mossman KD, Starr T, Dustin ML, Groves JT 2008. T cell receptor microcluster transport through molecular mazes reveals mechanism of translocation. Biophys J 94: 3286-3292.

Ding Y-H, Baker BM, Garboczi DN, Biddison WE, Wiley DC. 1999. Four A6-TCR/Peptide/HLA-A2 structures that generate very different $\mathrm{T}$ cell signals are nearly identical. Immunity 11: 45-56.

Douglass AD, Vale RD. 2005. Single-molecule microscopy reveals plasma membrane microdomains created by protein-protein networks that exclude or trap signaling molecules in T cells. Cell 121: 937-950.

Dushek O, Mueller S, Soubies S, Depoil D, Caramalho I, Coombs D, Valitutti S. 2008. Effects of intracellular calcium and actin cytoskeleton on TCR mobility measured by fluorescence recovery. PLoS ONE 3: e3913.

Ehrlich LIR, Ebert PJR, Krummel MF, Weiss A, Davis MM 2002. Dynamics of p56lck translocation to the T cell immunological synapse following agonist and antagonist stimulation. Immunity 17: 809-822.

Freiberg BA, Kupfer H, Maslanik W, Delli J, Kappler J, Zaller DM, Kupfer A. 2002. Staging and resetting T cell activation in SMACs. Nat Immunol 3: 911-917.

Gomez TS, Kumar K, Medeiros RB, Shimizu Y, Leibson PJ, Billadeau DD. 2007. Formins Regulate the actin-related protein $2 / 3$ complex-independent polarization of the centrosome to the immunological synapse. Immunity 26: $177-190$.

Gomez TS, McCarney SD, Carrizosa E, Labno CM, Comiskey EO, Nolz JC, Zhu P, Freedman BD, Clark MR, Rawlings DJ, et al. 2006. HS1 Functions as an Essential Actin-Regulatory Adaptor Protein at the Immune Synapse. Immunity 24: 741-752.

Grakoui A, Bromley SK, Sumen C, Davis MM, Shaw AS, Allen PM, Dustin ML. 1999. The immunological synapse: a molecular machine controlling $\mathrm{T}$ cell activation. Science 285: 221-227.

Gunzer M, Schäfer A, Borgmann S, Grabbe S, Zänker KS, Bröcker E-B, Kämpgen E, Friedl P. 2000. Antigen presentation in extracellular matrix: Interactions of $\mathrm{T}$ cells with dendritic cells are dynamic, short lived, and sequential. Immunity 13: 323-332.

Hartman NaC, Nye JA, Groves JT. 2009. Cluster size regulates protein sorting in the immunological synapse. Proc Natl Acad Sci 106: 12729-12734.

Henson JH, Svitkina TM, Burns AR, Hughes HE, MacPartland KJ, Nazarian R, Borisy GG. 1999. Two components of actin-based retrograde flow in sea urchin coelomocytes. Mol Biol Cell 10: 4075-4090.

Huppa JB, Axmann M, Mortelmaier MA, Lillemeier BF, Newell EW, Brameshuber M, Klein LO, Schutz GJ, Davis MM. 2010. TCR-peptide-MHC interactions in situ show accelerated kinetics and increased affinity. Nature 463: 963-967.

Ilani T, Vasiliver-Shamis G, Vardhana S, Bretscher A, Dustin ML. 2009. T cell antigen receptor signaling and immunological synapse stability require myosin IIA. Nat Immunol 10: $531-539$.

Irvine DJ, Purbhoo MA, Krogsgaard M, Davis MM. 2002. Direct observation of ligand recognition by $\mathrm{T}$ cells. Nature 419: 845-849.

Jacobelli J, Bennett FC, Pandurangi P, Tooley AJ, Krummel MF. 2009. Myosin-IIA and ICAM-1 regulate the interchange between two distinct modes of T cell migration. J Immunol 182: 2041-2050.

Jacobelli J, Chmura SA, Buxton DB, Davis MM, Krummel MF. 2004. A single class II myosin modulates T cell motility and stopping, but not synapse formation. Nat Immunol 5: 531-538.

Jenkins MR, Tsun A, Stinchcombe JC, Griffiths GM. 2009. The strength of $\mathrm{T}$ cell receptor signal controls the polarization of cytotoxic machinery to the immunological synapse. Immunity 31: 621-631.

Kaizuka Y, Douglass AD, Vardhana S, Dustin ML, Vale RD. 2009. The coreceptor CD2 uses plasma membrane microdomains to transduce signals in T cells. J Cell Biol 185: $521-534$

Kaizuka Y, Douglass AD, Varma R, Dustin ML, Vale RD. 2007. Mechanisms for segregating $T$ cell receptor and adhesion molecules during immunological synapse formation in Jurkat T cells. Proc Natl Acad Sci 104: $20296-$ 20301.

Kim ST, Takeuchi K, Sun Z-YJ, Touma M, Castro CE, Fahmy A, Lang MJ, Wagner G, Reinherz EL. 2009. The $\alpha \beta$ T cell receptor is an anisotropic mechanosensor. J Biol Chem 284: 31028-31037.

Krogsgaard M, Li Q-j, Sumen C, Huppa JB, Huse M, Davis MM. 2005. Agonist/endogenous peptide-MHC heterodimers drive $\mathrm{T}$ cell activation and sensitivity. Nature 434: $238-243$.

Krogsgaard M, Prado N, Adams EJ, He X-1, Chow D-C, Wilson DB, Garcia KC, Davis MM. 2003. Evidence that structural rearrangements and/or flexibility during TCR binding can contribute to T cell activation. Molecular Cell 12: $1367-1378$.

Krummel MF, Sjaastad MD, Wulfing C, Davis MM. 2000. Differential clustering of CD4 and CD3zeta during T cell recognition. Science 289: 1349-1352.

Lanzavecchia A, Iezzi G, Viola A. 1999. From TCR engagement to $\mathrm{T}$ cell activation: a kinetic view of $\mathrm{T}$ cell behavior. cell 96: $1-4$. 
Leitenberg D, Novak T, Farber D, Smith B, Bottomly K. 1996. The extracellular domain of CD45 controls association with the CD4-T cell receptor complex and the response to antigen-specific stimulation. J Exp Med 183: 249-259.

Lillemeier BF, Mortelmaier MA, Forstner MB, Huppa JB, Groves JT, Davis MM. 2010. TCR and Lat are expressed on separate protein islands on T cell membranes and concatenate during activation. Nat Immunol 11: 90-96.

Lillemeier BF, Pfeiffer JR, Surviladze Z, Wilson BS, Davis MM. 2006. Plasma membrane-associated proteins are clustered into islands attached to the cytoskeleton. Proc Natl Acad Sci 103: 18992-18997.

Ma Z, Sharp KA, Janmey PA, Finkel TH. 2008. Surfaceanchored monomeric agonist pMHCs alone trigger TCR with high sensitivity. PLoS Biology 6: e43.

McKeithan TW. 1995. Kinetic proofreading in T-cell receptor signal transduction. Proc Natl Acad Sci 92: 5042 5046.

Mempel TR, Henrickson SE, von Andrian UH. 2004. T-cell priming by dendriticcells in lymph nodes occurs in three distinct phases. Nature 427: 154-159.

Miletic AV, Graham DB, Sakata-Sogawa K, Hiroshima M, Hamann MJ, Cemerski S, Kloeppel T, Billadeau DD, Kanagawa O, Tokunaga M, et al. 2009. Vav links the T cell antigen receptor to the actin cytoskeleton and $\mathrm{T}$ cell activation independently of intrinsic guanine nucleotide exchange activity. PLoS ONE 4: e6599.

Miletic AV, Sakata-Sogawa K, Hiroshima M, Hamann MJ, Gomez TS, Ota N, Kloeppel T, Kanagawa O, Tokunaga M, Billadeau DD, et al. 2006. Vav1 acidic region tyrosine 174 is required for the formation of $\mathrm{T}$ cell receptorinduced microclusters and is essential in T cell development and activation. J Biol Chem 281: 38257-38265.

Miller MJ, Wei SH, Parker I, Cahalan MD. 2002. Twophoton imaging of lymphocyte motility and antigen response in intact lymph node. Science 296: 1869-1873.

Monks CRF, Freiberg BA, Kupfer H, Sciaky N, Kupfer A. 1998. Three-dimensional segregation of supramolecular activation clusters in T cells. Nature 395: 82-86.

Mossman KD, Campi G, Groves JT, Dustin ML. 2005. Altered TCR signaling from geometrically repatterned immunological synapses. Science 310: 1191-1193.

Negulescu PA, Krasieva TB, Khan A, Kerschbaum HH, Cahalan MD. 1996. Polarity of T cell shape, motility, and sensitivity to antigen. Immunity 4: 421-430.

Nguyen K, Sylvain NR, Bunnell SC. 2008. T cell costimulation via the integrin VLA-4 inhibits the actin-dependent centralization of signaling microclusters containing the adaptor SLP-76. Immunity 28: 810-821.

Phee H, Abraham RT, Weiss A. 2005. Dynamic recruitment of PAK1 to the immunological synapse is mediated by PIX independently of SLP-76 and Vav1. Nat Immunol 6: $608-617$.

Pollard TD, Borisy GG. 2003. Cellular motility driven by assembly and disassembly of actin filaments. Cell 112: $453-465$.

Porter JC, Bracke M, Smith A, Davies D, Hogg N. 2002. Signaling through integrin LFA-1 leads to filamentous actin polymerization and remodeling, resulting in enhanced T cell adhesion. J Immunol 168: 6330-6335.
Mediation of T-Cell Activation by Actin Meshworks

Rozdzial MM, Malissen B, Finkel TH. 1995. Tyrosinephosphorylated $\mathrm{T}$ cell receptor zeta chain associates with the actin cytoskeleton upon activation of mature $\mathrm{T}$ lymphocytes. Immunity 3: 623-633.

Salojin KV, Zhang J, Delovitch TL. 1999. TCR and CD28 are coupled via ZAP-70 to the activation of the Vav/Rac-1-/ PAK-1/p38 MAPK signaling pathway. J Immunol 163: 844-853.

Shenoi H, Seavitt J, Zheleznyak A, Thomas ML, Brown EJ. 1999. Regulation of integrin-mediated T cell adhesion by the transmembrane protein tyrosine phosphatase CD45. J Immunol 162: 7120-7127.

Shulman Z, Shinder V, Klein E, Grabovsky V, Yeger O, Geron E, Montresor A, Bolomini-Vittori M, Feigelson SW, Kirchhausen T, et al. 2009. Lymphocyte crawling and transendothelial migration require chemokine triggering of high-affinity LFA-1 integrin. Immunity 30: 384-396.

Stefanova I, Hemmer B, Vergelli M, Martin R, Biddison WE, Germain RN. 2003. TCR ligand discrimination is enforced by competing ERK positive and SHP-1 negative feedback pathways. Nat Immunol 4: 248-254.

Stinchcombe JC, Majorovits E, Bossi G, Fuller S, Griffiths GM. 2006. Centrosome polarization delivers secretory granules to the immunological synapse. Nature 443: $462-$ 465.

Stone J, Conroy L, Byth K, Hederer R, Howlett S, Takemoto Y, Holmes N, Alexander D. 1997. Aberrant TCR-mediated signaling in CD45-null thymocytes involves dysfunctional regulation of Lck, Fyn, TCR-zeta, and ZAP-70. J Immunol 158: 5773-5782.

Suzuki J-i, Yamasaki S, Wu J, Koretzky GA, Saito T. 2007. The actin cloud induced by LFA-1-mediated outside-in signals lowers the threshold for T-cell activation. Blood 109: $168-175$.

Sykulev Y, Joo M, Vturina I, Tsomides TJ, Eisen HN. 1996. Evidence that a single peptide MHC complex on a target cell can elicit a cytolytic $\mathrm{T}$ cell response. Immunity 4: $565-571$.

Thomas ML, Brown EJ. 1999. Positive and negative regulation of Src-family membrane kinases by CD45. Immunol Today 20: 406-411.

Trowbridge IS, Thomas ML. 2003. CD45: An emerging role as a protein tyrosine phosphatase required for lymphocyte activation and development. Ann Rev Immunol 12: 85-116.

Tskvitaria-Fuller I, Seth A, Mistry N, Gu H, Rosen MK, Wulfing C. 2006. Specific patterns of Cdc42 activity are related to distinct elements of T cell polarization. J Immunol 177: 1708-1720.

Valensin S, Paccani Silvia R, Ulivieri C, Mercati D, Pacini S, Patrussi L, Hirst T, Lupetti P, Baldari Cosima T. 2002. F-actin dynamics control segregation of the TCR signaling cascade to clustered lipid rafts. Eur J Immunol 32: $435-446$.

Valitutti S, Muller S, Cella M, Padovan E, Lanzavecchia A. 1995. Serial triggering of many T-cell receptors by a few peptideÂ-MHC complexes. Nature 375: 148-151.

van der Merwe PA, Davis SJ, Shaw AS, Dustin ML. 2000. Cytoskeletal polarization and redistribution of cellsurface molecules during $\mathrm{T}$ cell antigen recognition. Seminars in Immunology 12: 5-21. 


\section{P. Beemiller and M.F. Krummel}

Varma R, Campi G, Yokosuka T, Saito T, Dustin ML. 2006. T cell receptor-proximal signals are sustained in peripheral microclusters and terminated in the central supramolecular activation cluster. Immunity 25: 117-127.

Wedagedera JR, Burroughs NJ. 2006. T-cell activation: A queuing theory analysis at low agonist density. Biophys $J$ 91: 1604-1618.

Willcox BE, Gao GF, Wyer JR, Ladbury JE, Bell JI, Jakobsen BK, van der Merwe PA. 1999. TCR Binding to peptideMHC stabilizes a flexible recognition interface. Immunity 10: $357-365$.

Wu J, Katzav S, Weiss A. 1995. A functional T-cell receptor signaling pathway is required for p95vav activity. Mol Cell Biol 15: 4337-4346.

Wulfing C, Sjaastad MD, Davis MM. 1998. Visualizing the dynamics of T cell activation: Intracellular adhesion molecule 1 migrates rapidly to the $\mathrm{T}$ cell/B cell interface and acts to sustain calcium levels. Proc Natl Acad Sci 95: 6302-6307.

Yokosuka T, Kobayashi W, Sakata-Sogawa K, Takamatsu M, Hashimoto-Tane A, Dustin ML, Tokunaga M, Saito T.
2008. Spatiotemporal regulation of $\mathrm{T}$ cell costimulation by TCR-CD28 microclusters and protein kinase C, Translocation. Immunity 29: 589-601.

Yokosuka T, Sakata-Sogawa K, Kobayashi W, Hiroshima M, Hashimoto-Tane A, Tokunaga M, Dustin ML, Saito T. 2005. Newly generated $T$ cell receptor microclusters initiate and sustain $\mathrm{T}$ cell activation by recruitment of Zap70 and SLP-76. Nat Immunol 6: 1253-1262.

Zeng R, Cannon JL, Abraham RT, Way M, Billadeau DD, Bubeck-Wardenberg J, Burkhardt JK. 2003. SLP76 Coordinates Nck-dependent Wiskott-Aldrich syndrome protein recruitment with Vav-1/Cdc42-Dependent Wiskott-Aldrich syndrome protein activation at the T Cell-APC contact site. J Immunol 171: 13601368.

Zhang J, Shehabeldin A, da Cruz LAG, Butler J, Somani A-K, McGavin M, Kozieradzki I, dos Santos AO, Nagy A, Grinstein S, et al. 1999. Antigen receptor-induced activation and cytoskeletal rearrangement are impaired in WiskottAldrich syndrome protein-deficient lymphocytes. J Exp Med 190: 1329-1342. 


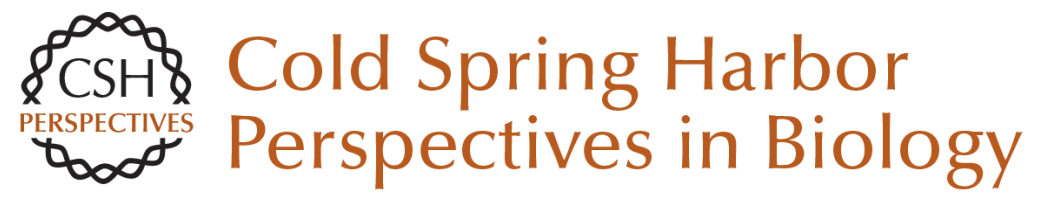

\section{Mediation of T-Cell Activation by Actin Meshworks}

Peter Beemiller and Matthew F. Krummel

Cold Spring Harb Perspect Biol 2010; doi: 10.1101/cshperspect.a002444 originally published online August 11, 2010

\section{Subject Collection Immunoreceptor Signaling}

The Coordination of T-cell Function by Serine/Threonine Kinases

David Finlay and Doreen Cantrell

ITAM-mediated Signaling by the T-Cell Antigen Receptor

Paul E. Love and Sandra M. Hayes

Coordination of Receptor Signaling in Multiple Hematopoietic Cell Lineages by the Adaptor

Protein SLP-76

Martha S. Jordan and Gary A. Koretzky

The Cytoskeleton Coordinates the Early Events of B-cell Activation

Naomi E. Harwood and Facundo D. Batista

An Enigmatic Tail of CD28 Signaling

Jonathan S. Boomer and Jonathan M. Green

\section{Mediation of T-Cell Activation by Actin Meshworks \\ Peter Beemiller and Matthew F. Krummel}

T-Cell Signaling Regulated by the Tec Family

Kinase, Itk

Amy H. Andreotti, Pamela L. Schwartzberg, Raji E. Joseph, et al.
Perspectives for Computer Modeling in the Study of $\mathrm{T}$ Cell Activation Jesse Coward, Ronald N. Germain and Grégoire Altan-Bonnet

Structural Biology of the T-cell Receptor: Insights into Receptor Assembly, Ligand Recognition, and Initiation of Signaling

Kai W. Wucherpfennig, Etienne Gagnon, Melissa J. Call, et al.

Src-family and Syk Kinases in Activating and Inhibitory Pathways in Innate Immune Cells:

Signaling Cross Talk Clifford A. Lowell

The LAT Story: A Tale of Cooperativity, Coordination, and Choreography

Lakshmi Balagopalan, Nathan P. Coussens, Eilon Sherman, et al.

Antigen Receptor Signaling to NF- $\mathrm{KB}$ via CARMA1, BCL10, and MALT1 Margot Thome, Jean Enno Charton, Christiane Pelzer, et al.

It's All About Change: The Antigen-driven Initiation of B-Cell Receptor Signaling Wanli Liu, Hae Won Sohn, Pavel Tolar, et al.

ZAP-70: An Essential Kinase in T-cell Signaling Haopeng Wang, Theresa A. Kadlecek, Byron B. Au-Yeung, et al.

For additional articles in this collection, see http://cshperspectives.cshlp.org/cgi/collection/

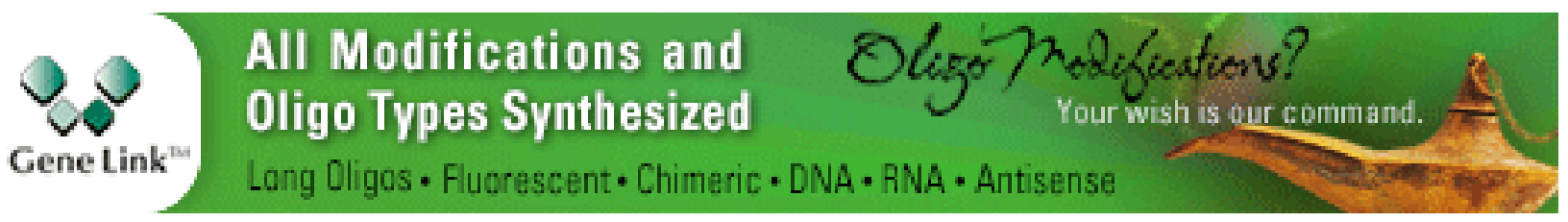




\section{Lipid Signaling in T-Cell Development and Function \\ Yina H. Huang and Karsten Sauer}

Understanding the Structure and Function of the Immunological Synapse

Michael L. Dustin, Arup K. Chakraborty and Andrey S. Shaw

For additional articles in this collection, see http://cshperspectives.cshlp.org/cgi/collection/

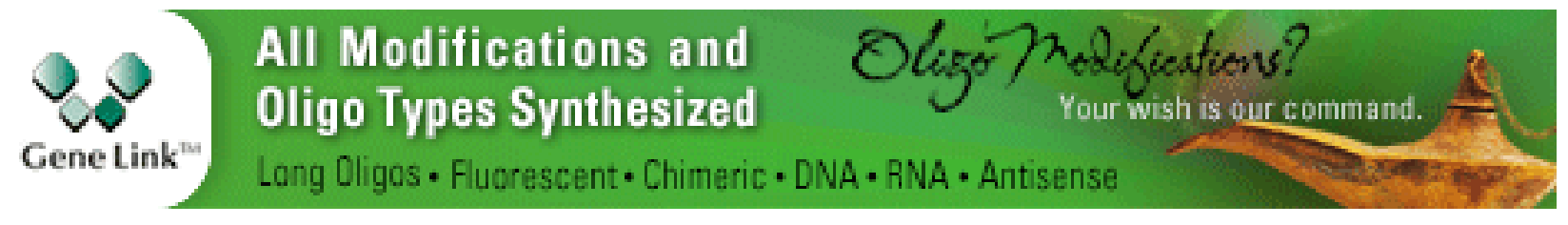

Copyright @ 2010 Cold Spring Harbor Laboratory Press; all rights reserved 